\title{
Reducción de pérdidas ocasionadas por flujos de dispersión en el tanque de transformadores de distribución
}

\author{
Héctor Cadavid $^{18}$, Juan A. Vergara ${ }^{2}$, Paúl C. González ${ }^{3}$ \\ 'Grupo de Investigación en Alta Tensión GRALTA, Universidad del Valle, Cali, Colombia, \\ ${ }^{1 s}$ hector.cadavid@correounivalle.edu.co \\ ${ }^{2}$ Grupo de Investigación en Alta Tensión GRALTA, Universidad del Valle, Cali, Colombia \\ jualfove@hotmail.com \\ ${ }^{3}$ Grupo de Investigación en Alta Tensión GRALTA, Universidad del Valle, Cali, Colombia \\ paul.acdc@gmail.com
}

\section{Resumen}

(Recibido: Mayo 7 de 2010 Aceptado: Mayo 3 de 2011)

En este artículo se examina la simulación, implementación y validación del método de pantalla magnética modoancho de longitud variable, como mecanismo para reducir las pérdidas ocasionadas por los flujos de dispersión en el tanque de un transformador de distribución monofásico de $15 \mathrm{KVA}$. Inicialmente a través de la simulación en 2D con el método de elementos finitos MEF, se estableció el comportamiento de las pérdidas en las paredes del tanque del transformador, con y sin pantalla magnética, y posteriormente, a través de pruebas adicionales de laboratorio, se realizó la respectiva verificación en el transformador. El análisis de los resultados obtenidos en esta investigación muestra que hay un impacto significativo en la reducción del nivel de pérdidas adicionales, con la implementación de la pantalla magnética y por lo tanto tal metodología de mitigación de pérdidas seleccionada es adecuada y viable técnica y económicamente.

Palabras clave: Pérdidas adicionales, Flujo de dispersión, Pantalla magnética, Corrientes Eddy, Método de elementos finitos MEF.

ELECTRICAL ENGINEERING

\section{Reduction of losses caused by leakage flux in the tank of distribution transformers}

\begin{abstract}
In this paper it was examined the simulation, implementation and validation of the method of magnetic width-size shunt of varying length, as mechanism to reduce the losses caused by the leakage flux in the tank of a single-phase distribution transformer of $15 \mathrm{KVA}$. Initially through the simulation in 2D with the Finite-Element Method FEM, the behavior of the losses settled down in the walls of the tank of the transformer, with and without magnetic screen, and later on, through additional tests of laboratory, it was carried out the respective verification in the transformer. The analysis of the results obtained in this investigation shows that there is a significant impact in the reduction of the level of stray losses, with the implementation of the magnetic shunt and therefore such a methodology of selected mitigation of losses is adapted and viable technical and economically.
\end{abstract}

Keywords: Stray losses, Leakage flux, Magnetic shunt, Eddy currents, Finite-Element Method FEM. 


\section{Introducción}

La eficiencia en los transformadores de distribución se ha incrementado gradualmente con los nuevos desarrollos tecnológicos en los materiales (Ushigami et al., 2003; Cunha \& Paolinelli, 2008) y en los métodos de fabricación (Georgilakis et al., 2001; Kefalas et al., 2008), alcanzando niveles típicos que oscilan entre el $95 \%$ y $99 \%$ o más. Aun así y como resultado de la gran cantidad de unidades instaladas (alrededor de 50 millones, solo en Estados Unidos), cerca de un tercio de las pérdidas totales en los sistemas eléctricos de potencia estan relacionadas con transformadores de distribución, lo que representa aproximadamente el $2 \%$ de la energía eléctrica total generada (Lebot, 2002). Por esta razón y amparados en las nuevas políticas ambientales a nivel mundial, en los últimos años se ha tornado de gran interés la reducción de pérdidas en los transformadores de distribución, tal como se hace en los transformadores de potencia.

Siendo la naturaleza del fenómeno idéntica para ambos casos, tradicionalmente el estudio de las pérdidas ha estado concentrado en el área de los transformadores potencia, los cuales acaparan la atención en alrededor del $52 \%$ de la mayoría de investigaciones reportadas en relación con solo un $17 \%$ para transformadores distribución (Olivares et al., 2009). Esta importante diferencia ha estado motivada principalmente por el tamaño de las unidades de distribución en comparación con las de potencia, ya que en la mayoría de los casos, no justifican una adecuada relación coste / beneficio que haga viable y factible la implementación de las estrategias para la reducción en pérdidas. Sin embargo, durante el transcurso de la última década se ha empezado a prestar mayor interés investigativo hacia el lado de los transformadores de distribución, planteando nuevas alternativas que hacen mucho más favorable la inversión en reducción de pérdidas.

Las investigaciones desarrolladas en este campo se han orientado al estudio y reducción de pérdidas ocasionadas por los flujos de dispersión denominadas como pérdidas adicionales (Olivares et al., 2002; Venegas et al., 2008), debido a que estas pérdidas representan entre el $3 \%$ y $20 \%$ de las pérdidas totales del transformador (Ras, 1991) y de las cuales, en el caso de las unidades de distribución, una significativa parte se disipa en las paredes del tanque del transformador (Olivares et al., 2003; Olivares et al., 2004).

El avance de programas computacionales que utilizan métodos numéricos (Amoiralis et al., 2008; Sykulski, 2009), tales como el método de elementos finitos MEF, así como la utilización de sistemas y algoritmos expertos (Amoiralis et al., 2009; Georgilakis, 2009), son gran ayuda para comprender y analizar los fenómenos físicos complejos como estos, calcular las pérdidas, evaluar e implementar un método definido.

\section{Métodos para la mitigación de pérdidas por flujo de dispersión}

Las pérdidas por flujo de dispersión tienen incidencia directa en los principales elementos constructivos del transformador tales como devanados, bordes del núcleo, viga retenedora del yugo ("Frames"), placa retenedora de pierna del transformador ("Tie plate" y "Flitch plate"), tanque y cualquier otro elemento constructivo con propiedades magnéticas. Para la reducción de pérdidas por flujos de dispersión se utilizan distintos métodos, los cuales cambian según el elemento constructivo del transformador. Para disminuir las pérdidas, en el caso específico del tanque o cuba del transformador, comúnmente son utilizados tres métodos de mitigación (Kulkarni, 2004):

-Desvíos o pantallas en el yugo, el cual confina el campo de dispersión que sale de los devanados con el propósito de presentar menor cantidad de flujo externo en el núcleo y los devanados.

- Pantallas o desvíos magnéticos en los componentes estructurales, las cuales brindan una ruta de baja reluctancia para el flujo de dispersión restringiendo esta ruta de un modo predeterminado. Un modelo ideal de pantalla magnética (permeabilidad infinita) no tiene diferencia de potencial magnético a través de su longitud. Si las pantallas magnéticas tienen un adecuado espesor y son construidas con 
laminaciones de acero al silicio de grano orientado (CRGO) con bajas características Watts/Kg, sus pérdidas son casi insignificantes. Generalmente, se dejan trozos de la laminación del núcleo para hacer las pantallas magnéticas. Básicamente hay dos tipos de pantallas magnéticas, pantallas en modo-ancho (paralelo) y modo-borde (vertical), tal como se ilustra en las Figuras 1 y 2. La pantalla modo-ancho fijo (más comúnmente usada) se ubica sobre el tanque como se muestra en la Figura 1a, y el espesor de las pantallas puede ser tan pequeño como sea posible con el propósito de reducir la entrada de pérdidas en su posición inferior y superior, donde el campo de dispersión penetra radialmente.

Debido a que la incidencia de flujo radial penetra en la pantalla en diferentes puntos (principalmente en los extremos) y que la densidad de flujo en la pantalla es máxima en el centro, se utiliza una pantalla magnética de longitud variable (Figura 1b). La mayor parte de incidencia de flujo puede llegar hasta las pantallas a través de sus delgados finales reduciendo así las entradas de pérdidas. Experimentalmente se ha reportado una mejor efectividad en la reducción del nivel de pérdidas adicionales con la utilización de la pantalla magnética modo borde en relación con la pantalla modo ancho (Yongbin et al., 1994), porque con la incidencia de flujo radial sobre el espesor (borde) de sus láminas resultan pérdidas Eddy insignificantes.
-Escudos de corriente Eddy, son utilizados escudos de aluminio o cobre para proteger componentes estructúrales de las altas corrientes y campos de dispersión. Las corrientes Eddy inducidas en ellos repelen la incidencia de flujo de dispersión reduciendo las pérdidas en componentes estructúrales. En la mayoría de los casos, las pérdidas en los componentes estructurales y el escudo de corrientes Eddy son mayores que las de los componentes estructurales y pantallas magnéticas. Sin embargo, los escudos de corrientes Eddy tienen la ventaja de ser maleables para su instalación y el peso es usualmente más bajo que el de los desvíos magnéticos. Para lograr un apantallamiento efectivo se requiere un espesor de entre 10 a 12 mm para los escudos fabricados en cobre y de 8 a $10 \mathrm{~mm}$ para los de aluminio (Olivares et al, 2002).

\section{Modelamiento y simulación}

El modelo del transformador utilizado, corresponde al de un transformador de distribución monofásico, implementado en 2D con el método de elementos finitos MEF (Vergara, 2008), a través del software comercial ANSYS 10.0 .

Las características generales de la unidad utilizada son las indicadas en la Tabla 1.

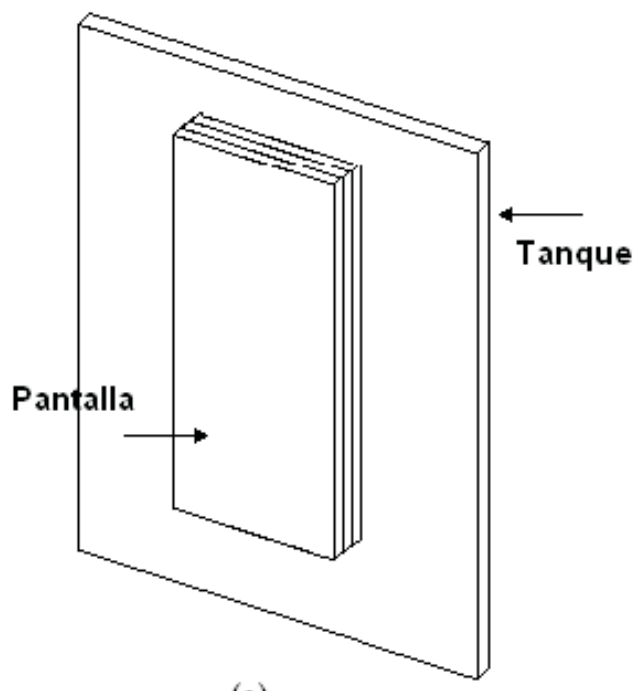

(a)

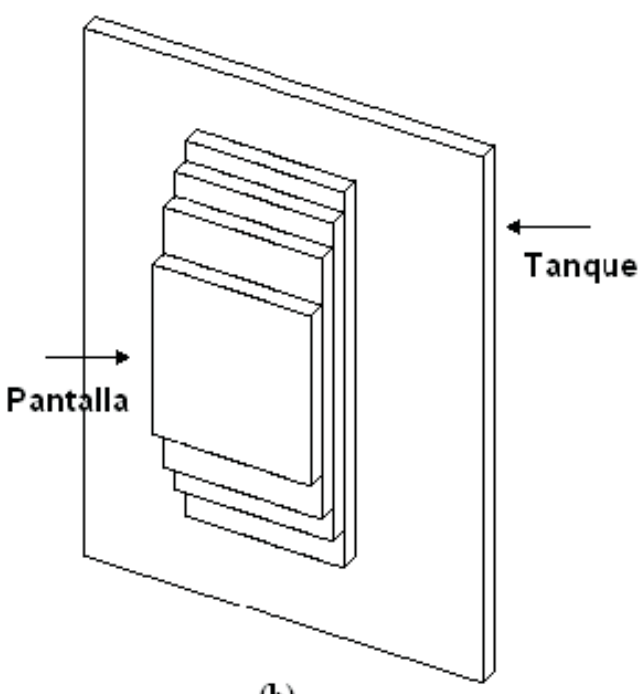

(b)

Figura 1. Tipos de pantallas magnéticas modo ancho: paralela fija (a) y paralela variable (b). 


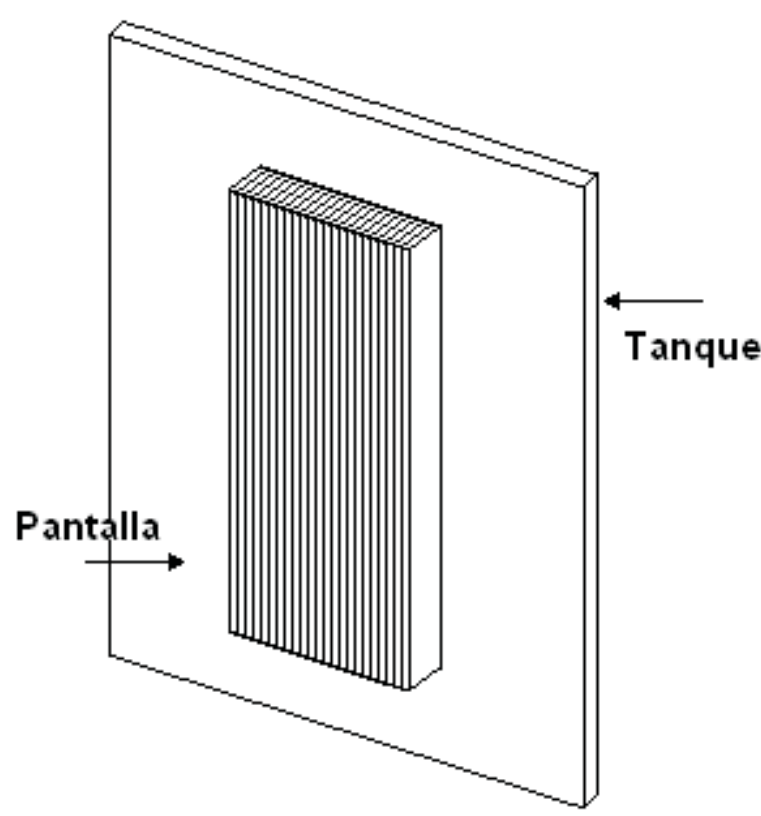

Figura 2. Pantalla magnética modo borde (vertical).

Tabla 1. Datos nominales transformador prototipo.

\begin{tabular}{lc}
\hline Parámetro & Valor Nominal \\
\hline Tipo & Distribución \\
Número de fases & 1 \\
Potencia (KVA) & 15 \\
Voltaje primario (V) & 13200 \\
Voltaje secundario (V) & $240 / 120$ \\
Corriente primaria (A) & 1.14 \\
Corriente secundaria (A) & 62.5 \\
\hline
\end{tabular}

El transformador de distribución se construye teniendo en cuenta las siguientes consideraciones geométricas básicas:

- Las cabezas de bobinas del transformador queden dentro del corte en 2D (Figura 3a).

- No se tiene en cuenta los canales refrigeración ubicados en las mitades de los devanados de alta y baja tensión del transformador.

-El núcleo es laminado pero se modela de forma maciza.

- Por su simetría, en la geometría solo se modela la mitad del corte transversal en 2D del

transformador, correspondiente a 1/4 del volumen total (Figura 3b).

La geometría se construyó utilizando el lenguaje código de ANSYS y a cada dimensión del transformador se le asignó un parámetro y junto a este, el valor de la medida, lo que permite variar las dimensiones de cada parte del transformador de forma más rápida, considerando que para implementar el método de disminución de pérdidas por flujo de dispersión (en este caso el de la pantalla magnética modo-ancho con longitud variable) se pueden modificar los parámetros $\mathrm{y}$ observar el comportamiento frente a tales variaciones. En la Figura 4 se muestra el modelo del transformador en dos dimensiones sin pantalla magnética.

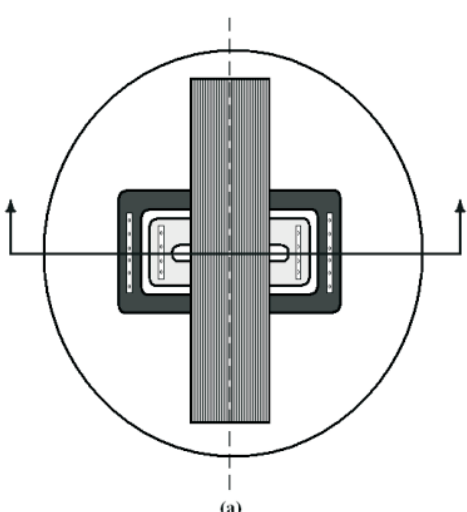

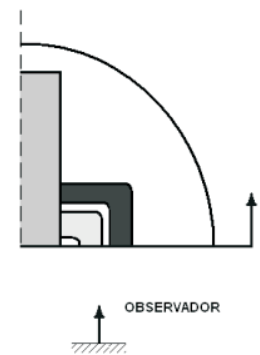

Figura 3. Cortes transversales modelo transformador 2D: vista superior (a) y mitad del corte (b).

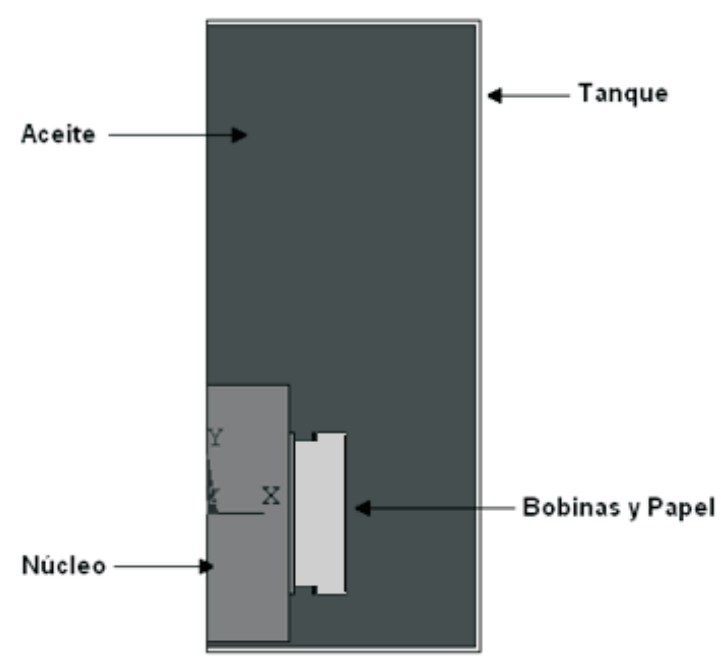

Figura 4. Vista transformador 2D sin pantalla magnéticaANSYS 10.0. 
En primera instancia y como referencia para el análisis posterior, se realiza la simulación del transformador sin pantalla magnética. Para ello se empleó el análisis armónico definiendo una frecuencia base de $60 \mathrm{~Hz}$ (asumiendo una corriente plenamente sinusoidal). La simulación se realizó bajo condición de cortocircuito a corriente nominal, para comparar las pérdidas resultantes, con las obtenidas en la pruebas de laboratorio. En la Figura 5a y 5b, se muestra la distribución de las líneas de flujo magnético obtenidas como resultado del proceso de simulación y donde se observa claramente que gran parte de las líneas que salen de las cabezas de bobinas entran en la parte lateral de la pared del tanque, igual que en la parte inferior. En la parte superior del tanque las líneas que se presentan por flujo de dispersión son mínimas.

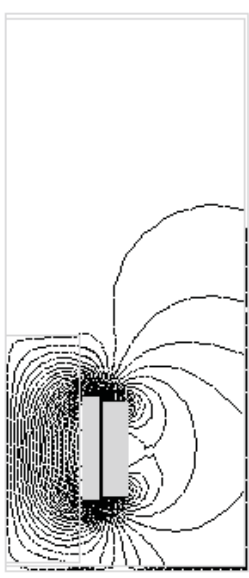

(a)

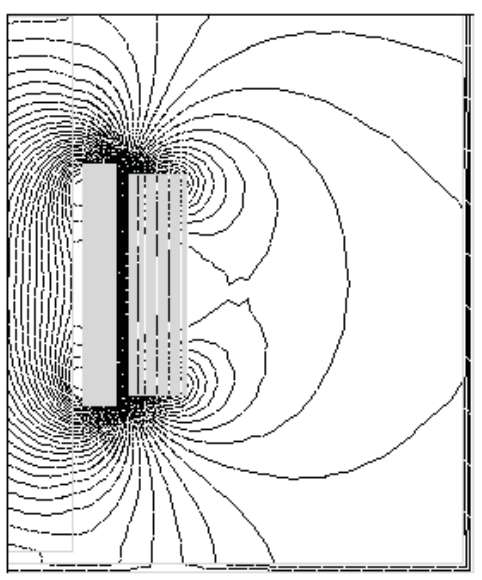

(b)
Figura 5. Distribución de las líneas del flujo magnético transformador sin pantalla magnética: modelo completo (a) y detalle en las cercanías a la pared del tanque (b).

En la Figura 6, se observa la distribución de la densidad de flujo magnético resultante y donde se puede apreciar que la mayor concentración en el tanque se presenta frente a los devanados. También se observa que el máximo valor de densidad de flujo se encuentra en canal de refrigeración entre alta-baja tensión y el mínimo valor en parte superior del tanque (en la tapa del tanque del transformador).

El método elegido para implementar la reducción de pérdidas por flujo de dispersión es el método de pantallas magnéticas en las paredes del tanque en modo-ancho con longitud variable, el cual se ha seleccionado por su fácil implementación comparada con los otros métodos de disminución de pérdidas discutidos anteriormente.

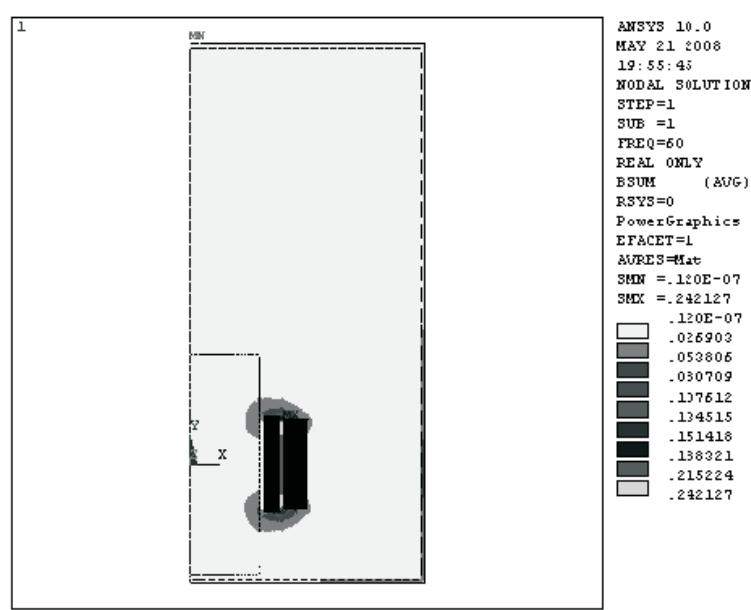

Figura 6. Distribución densidad de flujo magnético transformador sin pantalla magnética.

La simulación de este método se inicia con un escalón como si fuera una pantalla simple y luego se varía el número de escalones, hasta 3 . La pantalla magnética, con tres escalones, se instala en las paredes del tanque frente a las cabezas de bobinas.

Todos los atributos, características y propiedades del material, con el cual está construida la pantalla magnética en la simulación son iguales a los del núcleo (Chapas de Acero M1 con 0,23 mm de espesor). La disposición geométrica de la pantalla se ilustra en la Figura 7 y sus dimensiones físicas se especifican en la Tabla 2. En la Figura 8 se muestra la distribución de líneas de flujo magnético en el transformador y en la Figura 9 se observa la distribución de la densidad de flujo magnético, para el caso de la pantalla magnética con tres escalones respectivamente.

Con los valores de densidad de flujo magnético (B) e intensidad de campo magnético $\mathbf{( H )}$ en la pared del tanque, se calculan las pérdidas a través del método de impedancia de superficie (Holland et al., 1992). Para el cálculo total de las pérdidas ocasionadas por el flujo de dispersión en la pared 
del tanque del transformador (teniendo en cuenta las características no-lineales del material), con el coeficiente de linealidad $\mathrm{a}_{1}=1.3$, la expresión está determinada por:

$$
P=a_{1} \iint_{S} \sqrt{\frac{\omega \mu}{2 \sigma}} H_{R M S}^{2} d s
$$

Donde para el material constructivo del tanque la permeabilidad es $\mu=100 \times 4 \times \times 10^{7} \mathrm{H} / \mathrm{m}$, la conductividad $\sigma=2 \times 1.03 \times 10^{-7} \mathrm{~S} / \mathrm{m}$ y la frecuencia angular $\omega=2 \times \times 60 \mathrm{Rad} / \mathrm{s}$.
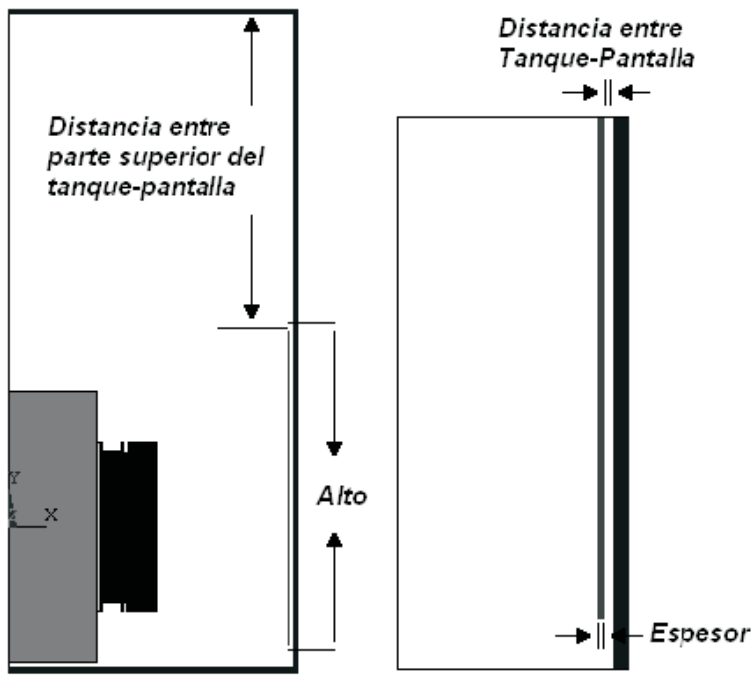

Figura 7. Disposición geométrica del apantallamiento magnético.

Tabla 2. Caracteristicas geométricas pantalla magnética hasta tres escalones.

\begin{tabular}{lccc}
\hline Dimensión & Primer & Segundo & Tercer \\
\hline & escalón & escalón & escalón \\
Altura $(\mathrm{cm})$ & 28.00 & 23.00 & 18.00 \\
Espesor $(\mathrm{cm})$ & 0.23 & 0.23 & 0.23 \\
$\begin{array}{l}\text { Distancia entre } \\
\text { tapa superior del }\end{array}$ & 24.45 & 23.95 & 21.45 \\
tanque -pantalla $(\mathrm{cm})$ & & & \\
\hline
\end{tabular}

Con los resultados obtenidos a través de la simulación para diferentes números de escalones en la pantalla, se procede a calcular las pérdidas en la pared del tanque, y se obtienen los respectivos resultados en la disminución de pérdidas (Tabla $3)$.

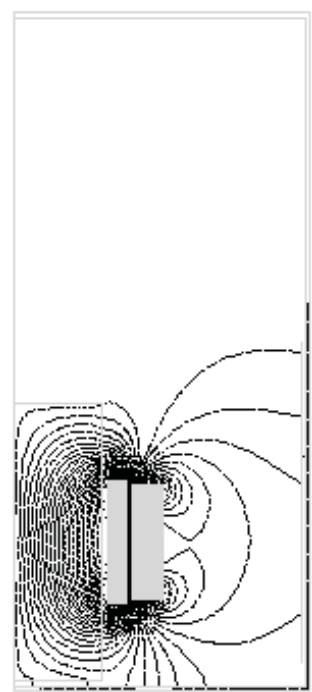

Figura 8. Distribución de las líneas de flujo magnético para transformador con pantalla de tres escalones.

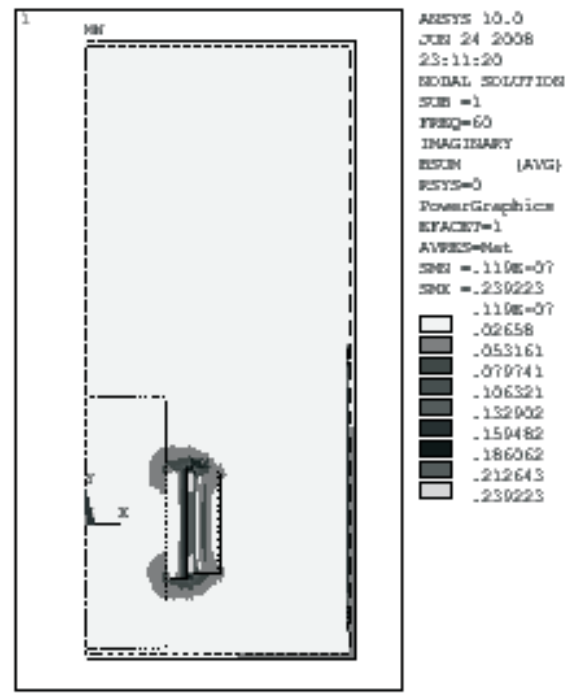

Figura 9. Distribución densidad de flujo magnético transformador con pantalla de tres escalones. 
Tabla 3. Resultados reducción de pérdidas en la pared del tanque del transformador según el modelo de elementos finitos $M E F$.

\begin{tabular}{lccc}
\hline & $\begin{array}{c}\text { Pérdidas } \\
\text { pared } \\
\text { del tanque } \\
\mathrm{P}(\mathrm{w})\end{array}$ & $\begin{array}{c}\text { Nivel } \\
\text { reducción } \\
\text { pérdidas } \\
\mathrm{P}(\mathrm{w})\end{array}$ & $\begin{array}{c}\text { Nivel } \\
\text { reducción } \\
\text { pérdidas } \\
(\%)\end{array}$ \\
\hline Sin pantalla & 8.57 & 0.00 & 0.00 \\
Pantalla(1 escalón) & 6.58 & 1.98 & 23.19 \\
Pantalla(2 escalones) & 5.14 & 3.43 & 40.02 \\
Pantalla(3 escalones) & 4.15 & 4.42 & 51.56 \\
\hline
\end{tabular}

\section{Metodología}

La validación del método consiste en implementar el método seleccionado para la disminución de pérdidas al transformador y luego hacer las pruebas correspondientes que determinen las pérdidas adicionales en el transformador. Con el resultado de los ensayos se podrá comparar en qué proporción se reducen las pérdidas en el transformador respecto a las pruebas realizadas sin el método implementado. Con esta comparación se podrá analizar la efectividad del método y si es fácil su implementación en el transformador. En la Figura 10 se muestra el transformador de distribución con el cual se realizan las pruebas.

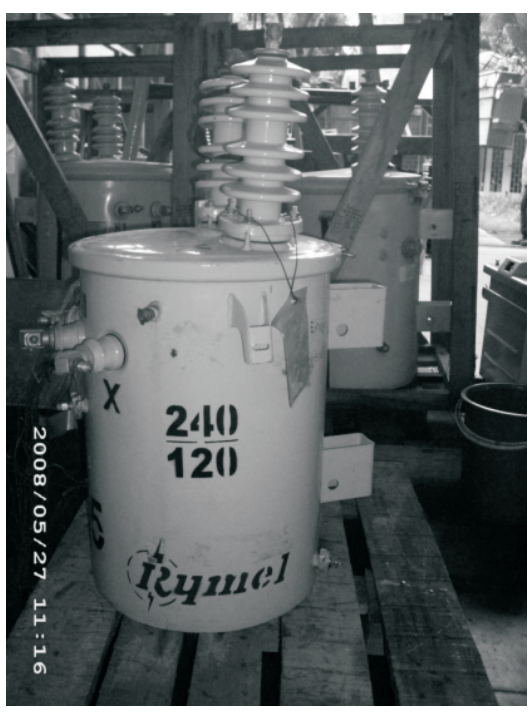

Figura 10. Transformador de prueba.
Las pantallas magnéticas a implementar se constituyen de láminas de acero al silicio de grano orientado (CRGO). El aislante (papel prensado) entre la pantalla y el tanque tiene un espesor de $3 \mathrm{~mm}$, medida que coincide con la distancia entre la pantalla y el tanque que se tomó para la simulación. En la instalación de las pantallas se debe tener en cuenta la dirección del grano orientado y la distancia entre el borde superior del transformador y la pantalla. Con estas consideraciones se instalan las dos pantallas en el transformador, las cuales son sujetadas provisionalmente con cuñas de madera entre el tanque y el núcleo tal como se muestra en las Figuras 11 y 12, para el caso de dos escalones. La madera no tiene ningún efecto magnético que pueda alterar el propósito del método reducción de pérdidas.

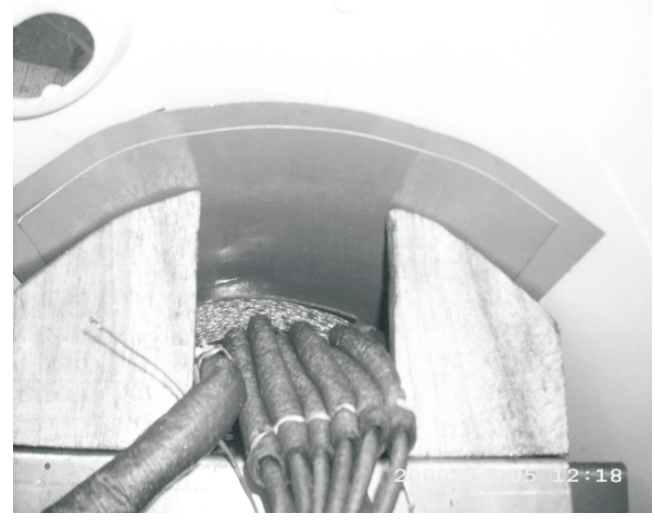

Figura 11. Detalle instalación pantalla magnética.

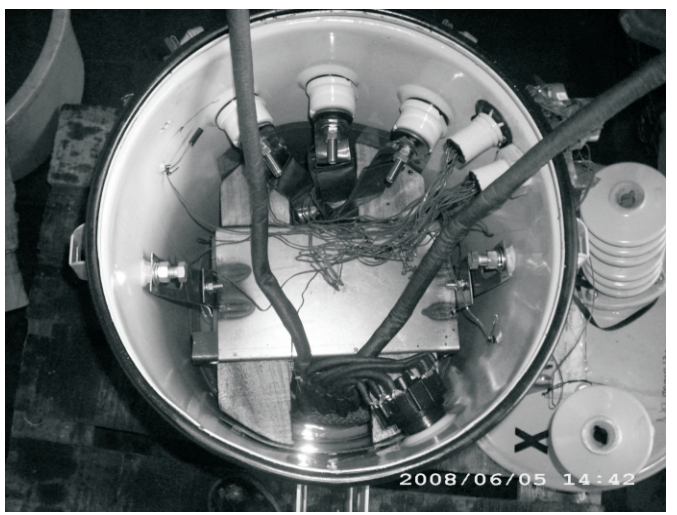

Figura 12. Aspecto final de instalación de la pantalla magnética en el transformador. 


\section{Resultados experimentales}

El transformador se sometió a los respectivos ensayos de rutina y los resultados de las pruebas de pérdidas se consignan en la Tabla 4 . Se puede apreciar y verificar una disminución en la magnitud de las pérdidas en la pared del tanque como consecuencia de la introducción del apantallamiento magnético al interior del transformador. Esto a su vez, es el resultado de la desviación de parte de las líneas de flujo de dispersión provenientes de los devanados, las cuales son recolectadas por la pantalla, evitando así que lleguen hasta el interior de la pared del tanque para de esta manera lograr una menor inducción de corrientes Eddy, lo cual corresponde con un menor nivel de pérdidas adicionales, tal como se explicó con anterioridad.

Tabla 4. Resultados reducción de pérdidas en la pared del tanque del transformador prototipo según pruebas experimentales.

\begin{tabular}{lccc}
\hline & $\begin{array}{c}\text { Pérdidas } \\
\text { adicionales } \\
\text { tanque } \\
\mathrm{P}(\mathrm{w})\end{array}$ & $\begin{array}{c}\text { Nivel } \\
\text { reducción } \\
\text { pérdidas } \\
\mathrm{P}(\mathrm{w})\end{array}$ & $\begin{array}{c}\text { Nivel } \\
\text { reducción } \\
\text { pérdidas } \\
(\%)\end{array}$ \\
\hline Sin pantalla & 8.57 & 0.00 & 0.00 \\
Pantalla (1 escalón) & 6.37 & 2.20 & 25.6 \\
Pantalla (2 escalones) & 4.66 & 3.91 & 45.5 \\
\hline
\end{tabular}

El nivel de reducción de pérdidas obtenido está directamente asociado con el número de escalones del apantallamiento, siendo mayor en la medida que existan varios escalones, ya que de esta forma es posible minimizar en mayor proporción, la incidencia del flujo de dispersión y las corrientes Eddy resultantes sobre la superficie del tanque, lo cual se retribuye en menores pérdidas adicionales.

En la Tabla 5 se muestra la comparación entre los resultados obtenidos experimentalmente y la simulación. Se puede apreciar la similitud entres ambos valores lo cual asegura la confiabilidad del modelo de elemento finitos, en la medida que representa con gran afinidad la realidad del modelo físico y el fenómeno de las pérdidas adicionales en el transformador. La diferencia entre los resultados calculados con los resultados medidos está en el rango de 12\% al 13\%. Esta diferencia se presenta por las propiedades no lineales del material y por las aproximaciones que se hacen en un modelo en 2D. Se puede implementar este método en un modelo en 3D y verificar su consistencia, pero ello requiere de un trabajo más dispendioso en la construcción de la geometría además de una mayor demanda en recursos y tiempo de procesamiento computacional.

Tabla 5. Comparación entre los resultados experimentales y los obtenidos por simulación.

\begin{tabular}{lccc}
\hline & $\begin{array}{c}\text { Reducción } \\
\text { pérdidas } \\
\text { validación } \\
\mathrm{P}(\mathrm{w})\end{array}$ & $\begin{array}{c}\text { Reducción } \\
\text { pérdidas } \\
\text { simulación } \\
\mathrm{P}(\mathrm{w})\end{array}$ & $\begin{array}{c}\text { Diferencia } \\
(\%)\end{array}$ \\
\hline Sin pantalla & 0.00 & 0.00 & 0.00 \\
Pantalla (1 escalón) & 2.20 & 1.98 & 9.6 \\
Pantalla (2 escalones) & 3.91 & 3.43 & 12.22 \\
\hline
\end{tabular}

\section{Conclusiones}

En la simulación se pudo comprobar el comportamiento magnético del método de mitigación (pantalla magnética modo-ancho de longitud variable), donde se observa claramente que la densidad de flujo en la pantalla se concentra en la mitad de la misma, justificando el ahorro de material en los extremos de la pantalla (longitud variable).

Con los resultados obtenidos a través de la simulación fue posible visualizar y verificar una reducción en las pérdidas adicionales en las paredes del tanque del transformador y cuantificar el impacto y la importancia de la variación en el número de escalones en la efectividad de la pantalla magnética. Con la validación del método en el laboratorio se confirman los porcentajes de reducción de pérdidas obtenidos en el cálculo y su efectividad.

\section{Agradecimientos}

Los autores quieren expresar agradecimientos a Colciencias, que a través del proyecto de investigación Cod. 110648925118 y a su programa de Jóvenes Investigadores. De igual manera a Rymel Ingeniería Eléctrica Ltda. 


\section{Referencias bibliográficas}

Amoiralis, E.I., Tsili, M.A. \& Kladas, A.G. (2009). Transformer Design and Optimization: A Literature Survey. IEEE Transactions on Power Delivery 24 (4), 1999-2024.

Amoiralis, E.I., Tsilli, M. A. \& Georgilakis, P.S. (2008). The state of the art in engineering methods for transformer design and optimization: a survey. Journal of Optoelectronics and Advanced Materials $10(5), 1149-1158$.

Cunha, M.A. \& Paolinelli, S.C. (2008). Low core loss non-oriented silicon steels. Journal of Magnetism and Magnetic Materials 320, 2485-2489.

Georgilakis, P.S. (2009). Spotlight on Modern Transformer Design. London, U.K.: SpringerVerlag London Limited.

Georgilakis, P.S., Doulamis, N.D., Doulamis, A.D., Hatziargyriou, N.D. \& Kollias, S. D. (2001). A novel iron loss reduction technique for distribution transformers based on a combined genetic algorithm - Neural network approach. IEEE Transactions on Systems Man and Cybernetics Part C-Applications and Reviews 31 (1), 16-34.

Holland, S.A., O'connell, G.P. \& Haydock, L. (1992). Calculating stray losses in power transformers using surface impedance with finite elements. IEEE Transactions on Magnetics 28 (2), 13551358.

Kefalas, T.D., Georgilakis, P.S., Kladas, A.G., Souflaris, A.T. \& Paparigas, D.G. (2008). Multiple grade lamination wound core: A novel technique for transformer iron loss minimization using simulated annealing with restarts and an anisotropy model. IEEE Transactions on Magnetics 44 (6), 1082-1085.

Kulkarni, S.V. \& Khaparde, S.A. (2004). Transformer Engineering, Desing and Practice. New York: Marcel-Dekker, Inc.
Lebot, B. (2002). Energy-Efficient Distribution Transformers: a Hidden Opportunity for Large Scale Energy Savings. International Energy Agency-OECD.

Olivares, J.C., Canedo, J., Moreno, P., Driesen, J., Escarela, R. \& Palanivasagam, S. (2002). Experimental study to reduce the distributiontransformers stray losses using electromagnetic shields. Electric Power Systems Research 63, 1-7.

Olivares, J.C., Escarela-Perez, R., Kulkarni, S.V., De Leon, F., Melgoza-Vasquez, E. \& HernandezAnaya, O. (2004). Improved insert geometry for reducing tank-wall losses in pad-mounted transformers. IEEE Transactions on Power Delivery 19(3), 1120-1126.

Olivares, J.C., Liu, Y.L., Canedo, J.M., EscarelaPerez, R., Driesen, J. \& Moreno, P. (2003). Reducing losses in distribution transformers. IEEE Transactions on Power Delivery 18 (3), 821-826.

Olivares-Galvan, J.C., Georgilakis, P.S. \& OconValdez, R. (2009). A Review of Transformer Losses. Electric Power Components and Systems 37 (9), 1046-1062.

Ras, E.O. (1991). Transformadores de potencia, de medida y de protección. Colombia: Alfaomega Marcombo.

Sykulski, J.K. (2009). Computational electromagnetics for design optimisation: the state of the art and conjectures for the future. Bulletin of the Polish Academy of Sciences-Technical Sciences 57 (2), 123-131.

Ushigami, Y., Mizokami, M., Fujikura, M., Kubota, T., Fujii, H. \& Murakami, K. (2003). Recent development of low-loss grain-oriented silicon steel. Journal of Magnetism and Magnetic Materials 254-255, 307-314. 